\title{
SIMULATION STUDY OF A MISSILE COLD LAUNCH SYSTEM
}

\author{
Robert GeęBocki, Mariusz Jacewicz \\ Warsaw University of Technology, Institute of Aeronautics and Applied Mechanics, Warsaw, Poland \\ e-mail: rgleb@meil.pw.edu.pl; mjacewicz@meil.pw.edu.pl
}

\begin{abstract}
In this paper the missile flight dynamics during the launch phase is studied. The main concept behind this work was to use a vertical cold launch system and the rapid pitch maneuver to achieve longer missile range and better firing coverage. A set of a small pulse rocket engines was used to obtain the desired missile attitude. The physical and mathematical models of the missile are described. The pulse jets control algorithm is presented. The computer program of the missile model has been developed in the Simulink environment. The missile behavior in the low-speed flight envelope has been examined. The results of numerical simulations in the form of the graphs are presented. It has been obtained that there exist several benefits of the cold launch method as increased range and higher target kill probability.
\end{abstract}

Keywords: guided missile, modeling, simulation

\section{Introduction}

In this paper, results of the preliminary design phase of a soft launch system for a ground-to-air missile are presented. The main objective of this work is to develop a general physical and mathematical model which may be used for missile flight simulation. The developed model can be used as a baseline for design, analysis and development of guided missiles. The common approach in the field of vertical launch is to use a hot launch technique when the missile main engine is started in the missile container. A potential problem associated with hot vertical launch is range loss due to the turnover maneuver and fuel consumption in the boost phase. This translates into reduced range and increased time to the target. A more sophisticated method is to use a cold launch, which could be complemented with a rapid pitch maneuver over the launcher. In this case, the missile should be equipped with a set of small pulse engines. At the beginning, the missile is ejected vertically upward from a missile canister with an initial velocity between of 15 to 35 meters per second without starting the main engine (Fig. 1).

When the missile is several meters over the ground, the first small engine is used to maintain the pitch angular velocity. Next, the second pulse engine is used to reduce the angular velocity again to zero. Finally, the main rocket engine is started and the object is flying toward the target. At least, two small jet engines are necessary to perform correctly the rapid pitch maneuver. Without using the second jet engine, the missile can rotate freely too much, and when the main engine is fired the missile may fall on the ground. It is desirable to start the main engine when the missile velocity is as low as possible. The missile may be ejected from the launch tube by a piston which can be driven by a compressed gas cylinder or by a pyrotechnic gas generator.

The main benefit of the vertical cold-launch method when compared with the hot-launch is its safety. The lower thermal signature of the launcher is achievable. In this case, there is no hot exhaust of the missile motor interaction with the launcher. The benefits of ejection launching are more and more important as missile size increases. This is the reason why the cold launch method is often used in the case of submarine-launched ballistic missiles. This type of launch can provide full 360 degrees of coverage in all launch sectors. It is possible to achieve longer range 


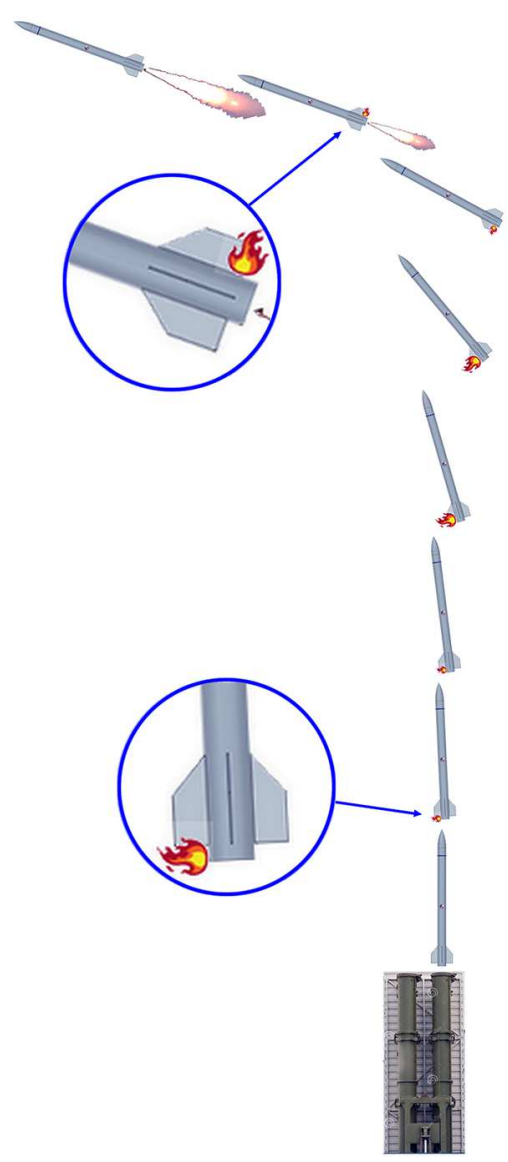

Fig. 1. Cold launch concept

because the main engine is not used in the launch phase and the fuel is consumed only in the midcourse and terminal phases (MBDA Systems, 2014). It offers also improved minimum range capability due to a more direct turnover trajectory that can enable earlier target acquisition. It is able to offer longer maximum range for a given mass when compared with the standard hot vertical launch. Unfortunately, there exist some problems with this type of launch. First of all, immediately after the launch, the missile is in an unstable phase of flight. Because of low velocity, the control surfaces are ineffective so the aerodynamic control at nearly zero speed is practically impossible. The rotational motion is weakly damped, which leads to problems with orienting of the missile in the demanded direction. The engines which control the attitude of the missile should has small delay, because there may appear problems with accuracy. The object after launch may fall on the launcher when the main engine fails. With the lack of a soft launch system there is no possibility of performing manoeuvres like the rapid pitch motion after the launch. There exists a lot of disturbances that can affect the missile trajectory during the launch phase. It is obvious that atmospheric conditions can vary in an unpredictable way. Wind is one of the most important factors which could disturb the missile motion. It is reasonable to assume that in strong wind conditions, the achieved missile pitch angle may be different from the desired one. Another factor that have impact on the launch procedure is the pressure in the missile container and launcher deflection. The reproducibility of the launch may be affected by several factors due to manufacturing inaccuracy (Fleeman, 2006).

\section{State of the art}

Nowadays, there exist only a few missiles which have both cold and pitch over launcher capabilities. One of the most known examples of this type of missiles is Common Anti-Air Modular 
Missile (Fig. 2) which was developed by the MBDA company and demonstrated in May 2011. CAMM has the minimum range under $1 \mathrm{~km}$ and the maximum range of about $25 \mathrm{~km}$. CAMM mass is $99 \mathrm{~kg}$, length $3.2 \mathrm{~m}$ and diameter $166 \mathrm{~mm}$ (MBDA Systems, 2014). The maximum speed of this missile is Mach 3. CAMM has folding tailfins and it is ejected from a compact canister tube by a piston at a height of $30 \mathrm{~m}$. The piston is retained within the tube so there is no launch debris. Next 8 small thrusters are used to point the missile at the target before the main motor fires. Missile turnover is achieved in time less than 1 second. Once turned over, the object is to be held at a selected heading and attitude by lateral thrusters.

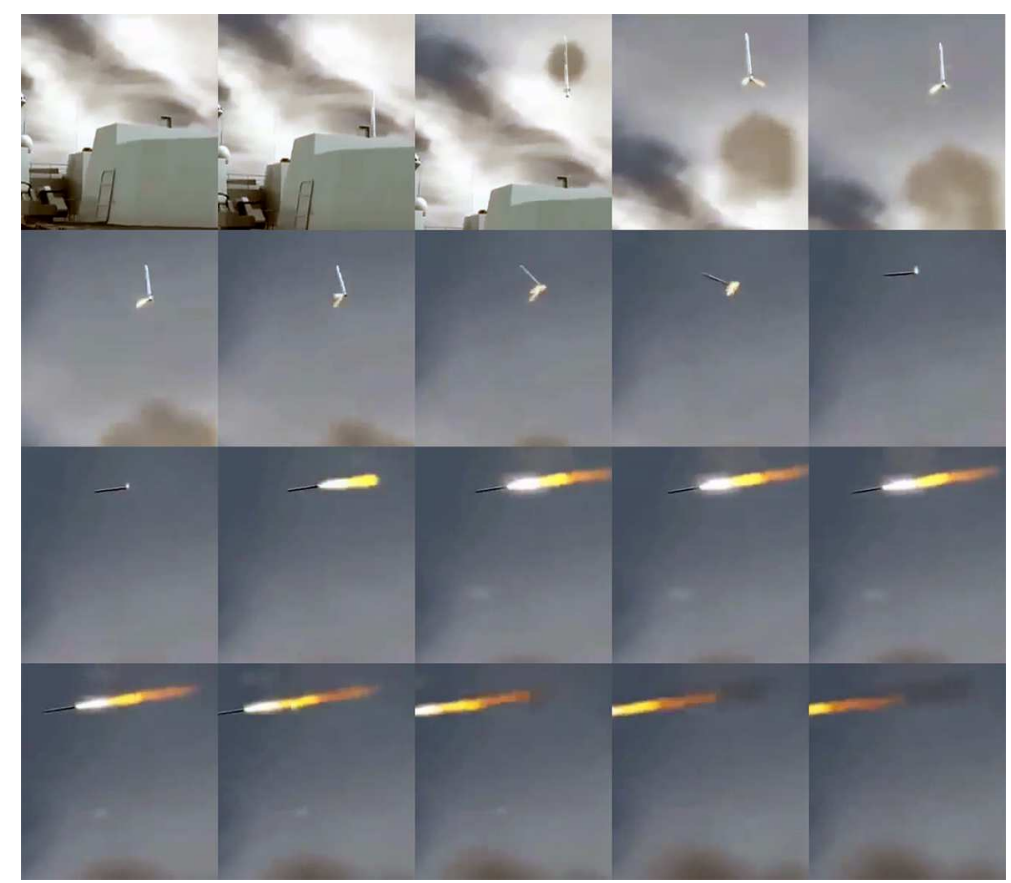

Fig. 2. CAMM missile launch (MBDA Systems, 2014)

CAMM is able to provide 360 degree coverage. Small thrusters are able to control the missile in all three planes. CAMM can be fired from the SYLVER and Mark 41 vertical launching systems or from Eurofighter Typhoon aircraft. There also exists CAMM-ER (extended range), and it has an additional booster which increases the missile range up to the value $45 \mathrm{~km}$. CAMM-ER is also bigger: 160 kilograms in weight, 4.2 meters in length, 190 millimeters in diameter. The CAMM launch platform is difficult to detect because of low acoustic, visual and thermal signature. This solution is safer for the ground staff, when compared to hot launch.

The second example of the air defense missile system in which the cold launch method is used is 9K330 Tor (SA-15 Gauntlet) (Fig. 3). The 9M331 missile weight is $167 \mathrm{~kg}$, diameter $235 \mathrm{~mm}$, length $2.9 \mathrm{~m}$, and it carries $15 \mathrm{~kg}$ warhead. Tor was developed in 1975 as a new version of Osa (SA-8 Gecko) surface-to-air missile system. Tor was designed for operation from very low to medium altitudes. It entered service with the Soviet Army in 1986, and Russian Army operates 172 of these systems. This system has good performance when used against aircraft (single missile destroy probability 26-75\%) and helicopters (50-88\%), but can also destroy modern targets like UAVs (85-95\%), precision guided munitions or cruise missiles (TOR-M1 9A331, 2012).

The system is mounted on the vehicle which is equipped with 8 missiles, associating radars and fire control systems. The combat vehicle can operate autonomously and the latest version can launch missiles even when the vehicle is on the move. The maximum range is between 5 and $12 \mathrm{~km}$ and the maximum altitude is $4-6 \mathrm{~km}$. The missiles have radio command guidance. The system can search for targets while on the move. Tor has reaction time of about 8-12 seconds from target detection to launch. There exist some versions of this system like Tor M, M1, M2K 


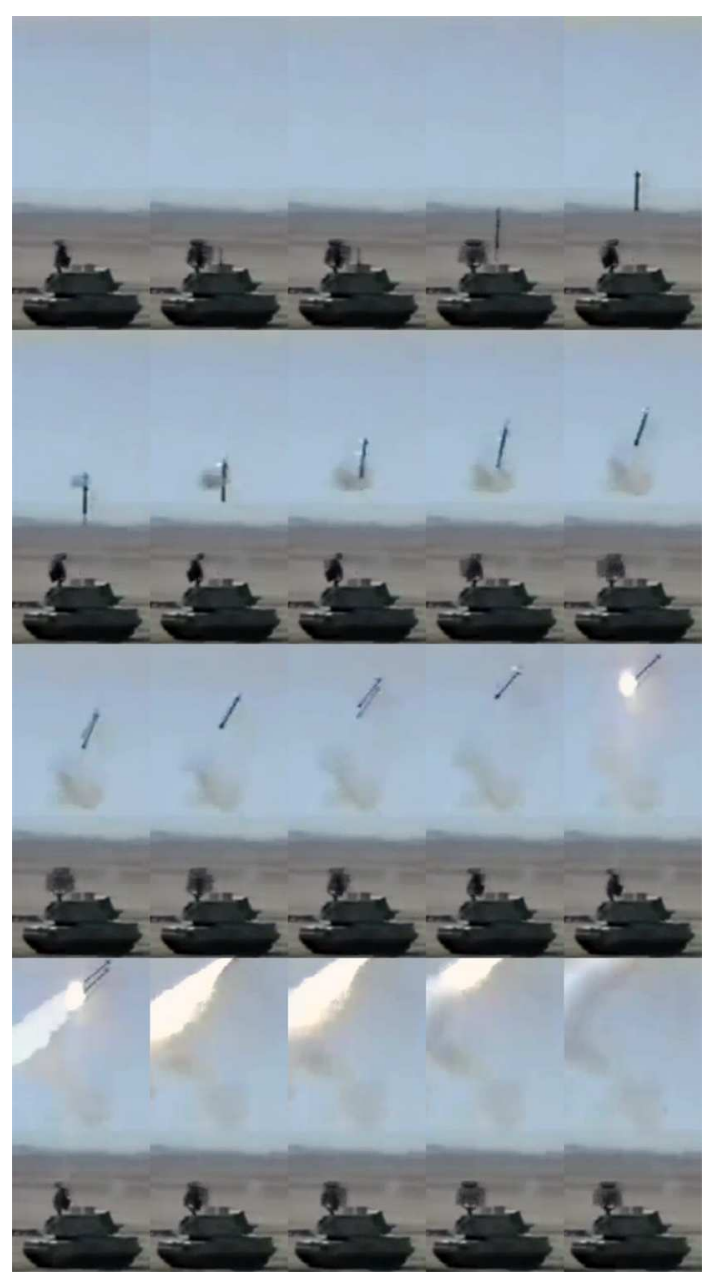

Fig. 3. Tor missile cold launch (TOR-M1 9A331, 2012)

or M2EK which are improved versions of the system. For example, Tor M1 can track up to 48 targets at the maximum range of $25 \mathrm{~km}$ and has higher kill probability.

\section{Physical model}

To analyze missile dynamics, it is assumed that the missile is a rigid body and has six degrees of freedom. This assumption, though strictly not valid for missiles with a high length-to-diameter ratio, is suitably accurate for describing missile motion during the launch phase. The total mass of the missile is assumed to be equal $58 \mathrm{~kg}$, length $2.3 \mathrm{~m}$, diameter $120 \mathrm{~mm}$ and fuel mass $24 \mathrm{~kg}$ (Fig. 4). The presented missile is smaller than the missiles presented in the "state of the art" Section. Values of these parameters are limited by the dimensions of the launcher.

It is assumed that the mass and moments of inertia change during the boost-phase. For short range, the flat Earth approximation is used. The vehicle aerodynamics is assumed to be nonlinear and quasi-steady. Moreover, it is assumed that the missile has two geometric and mass symmetry planes and the main motor thrust component passes through the missile center of gravity and is parallel to the missile longitudinal axis of symmetry. The reaction control system (RCS) uses twelve solid propellant pulse engines mounted, which generate the thrust directed normally to the main axis of symmetry of the object. The RCS is located $70 \mathrm{~mm}$ from the tail. Each small engine can be fired only once. 


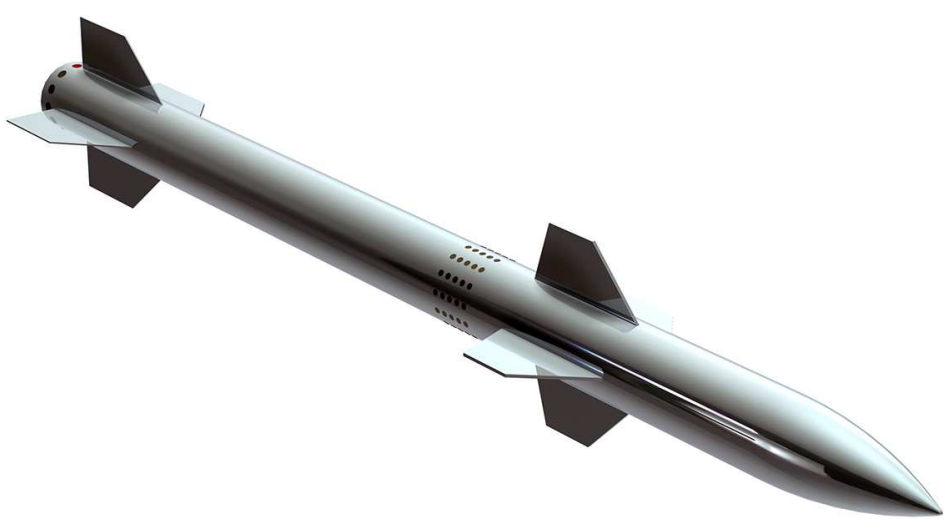

Fig. 4. 3D CAD missile model

\section{Mathematical model}

In this Section, the missile mathematical model six is derived. The basic frames used in this work are the inertial, body and gravity coordinate systems. The origin $O_{n}$ of the inertial coordinate system $O_{n} x_{n} y_{n} z_{n}$ is placed in any selected point on the Earth surface (Fig. 5). The axes $O_{n} x_{n}$
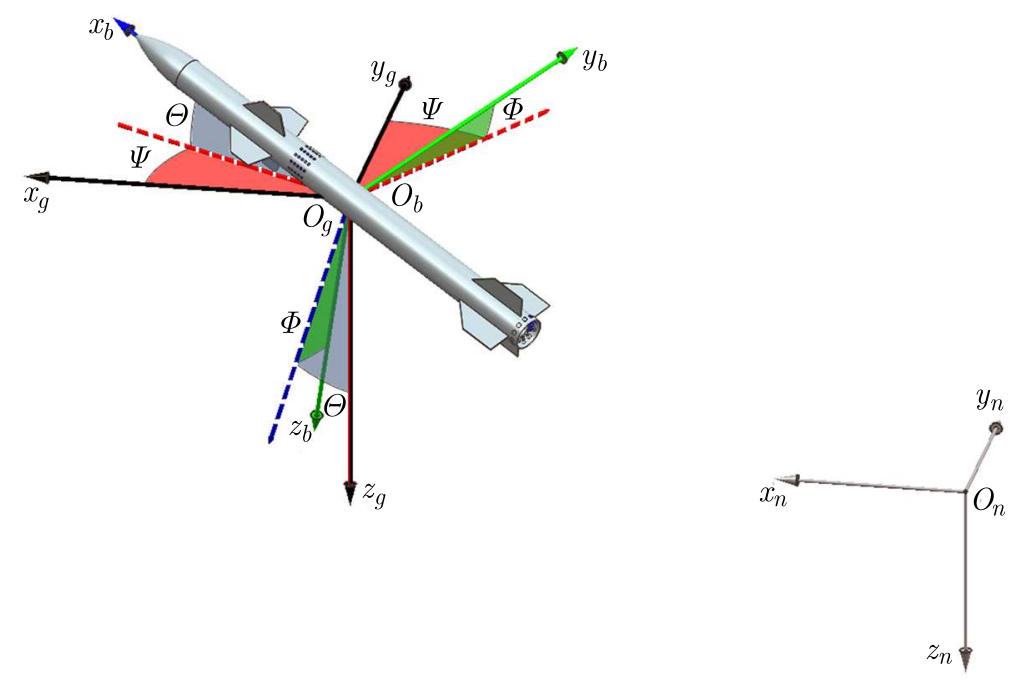

Fig. 5. Coordinate systems which have been used in simulation

and $O_{n} y_{n}$ lie in the plane which is perpendicular to the direction of gravity acceleration. The $O_{n} x_{n}$ is pointing north, and the $O_{n} y_{n}$ axis points east. The $O_{n} z_{n}$ axis is pointed vertically, it coincides with the direction of the Earth gravity acceleration. The missile equations of motion are derived in the conventional body coordinate system $O_{b} x_{b} y_{b} z_{b}$ (Blakelock, 1991). In the general case, the origin $O_{b}$ of this frame might be not at the center of gravity of the missile and moves forward as the after main engine burnout. In this article, it is assumed that the origin $O_{b}$ of this frame is coincident with the center of gravity of the missile. In the body coordinate system, the positive $O_{b} x_{b}$ axis coincides with the center line of the missile and it is designated as the roll-axis. The positive $O_{b} y_{b}$ axis is to the right of the $O_{b} x_{b}$ axis in the horizontal plane and it is designated as the pitch axis. The positive $O_{b} z_{b}$ axis points downward and it is designed as the yaw axis. The body axis system is fixed with respect to the missile and moves with the missile (Weinacht, 2004). The $O_{g} x_{g} y_{g} z_{g}$ is the gravity coordinate system. The centre $O_{g}$ of the gravity system of coordinates coincides with the centre of mass. The axis of the gravity system is parallel to the axis of the ground coordinate system. The mathematical model describing motion of the 
missile consists of six rigid-body degrees of freedom. The object orientation is parametrized using quaternions. Yaw, pitch, and roll angles are used only to visualize the results.

The system of six dynamic equations of motion is given by

$$
\mathbf{A} \dot{\mathbf{x}}+\mathbf{\Omega A x}=\left[\begin{array}{ll}
\mathbf{F}_{b} & \mathbf{M}_{b}
\end{array}\right]^{\mathrm{T}}
$$

where the missile state vector is

$$
\mathbf{x}=[U, V, W, P, Q, R]^{\mathrm{T}}
$$

and

$$
\mathbf{F}_{b}=\left[X_{b}, Y_{b}, Z_{b}\right]^{\mathrm{T}} \quad \mathbf{M}_{b}=\left[L_{b}, M_{b}, N_{b}\right]^{\mathrm{T}}
$$

where $U, V, W$ are linear velocities, $P, Q, R$ - angular velocities, $X_{b}, Y_{b}, Z_{b}$ - axial, side and normal forces along the body axes coordinate system. In a similar way, $L_{b}, M_{b}, N_{b}$ are rolling, pitching and yawing moments. The left-hand side of the equation describes the inertia loads in the missile frame of reference. In the most general case, when the missile centre of mass is not coincident with $O_{b}$, the inertia matrix is defined as follows (Żugaj and Głębocki, 2010)

$$
\mathbf{A}=\left[\begin{array}{cccccc}
m & 0 & 0 & 0 & S_{z} & -S_{y} \\
0 & m & 0 & -S_{z} & 0 & S_{x} \\
0 & 0 & m & S_{y} & -S_{x} & 0 \\
0 & -S_{z} & S_{y} & I_{x} & -I_{x y} & -I_{x z} \\
S_{z} & 0 & -S_{x} & -I_{y x} & I_{y} & -y z \\
-S_{y} & S_{x} & 0 & -I_{z x} & -I_{z y} & I_{z}
\end{array}\right]
$$

and the velocity matrix is

$$
\boldsymbol{\Omega}=\left[\begin{array}{cccccc}
0 & -R & Q & 0 & 0 & 0 \\
R & 0 & -P & 0 & 0 & 0 \\
-Q & P & 0 & 0 & 0 & 0 \\
0 & -W & V & 0 & -R & Q \\
W & 0 & -U & R & 0 & -P \\
-V & U & 0 & -Q & P & 0
\end{array}\right]
$$

where $m$ is the missile mass, $S_{x}, S_{y}, S_{z}$ - static moments, $I_{x}, I_{y}, I_{z}$ - moments of inertia, $I_{x y}, I_{x z}$, $I_{y z}$ - products of inertia.

The set of 6 scalar equations describing translational and rotational motion of the missile has the form

$$
\begin{aligned}
& m(\dot{U}+W Q-V R)-S_{x}\left(Q^{2}+R^{2}\right)+S_{y}(P Q-\dot{R})+S_{z}(P R+\dot{Q})=X_{b} \\
& m(\dot{V}+U R-W P)+S_{x}(P Q+\dot{R})-S_{y}\left(P^{2}+R^{2}\right)+S_{z}(Q R-\dot{P})=Y_{b} \\
& m(\dot{W}+V P-U Q)+S_{x}(P R-\dot{Q})+S_{y}(R Q+\dot{P})-S_{z}\left(P^{2}+Q^{2}\right)=Z_{b}
\end{aligned}
$$

and

$$
\begin{aligned}
& I_{x} \dot{P}-\left(I_{y}-I_{z}\right) R Q+I_{x y}(P R-\dot{Q})-I_{x z}(P Q+\dot{R})+I_{y z}\left(R^{2}-Q^{2}\right) \\
& \quad+S_{y}(\dot{W}+V P-U Q)-S_{z}(\dot{V}+U R-W P)=L_{b} \\
& I_{y} \dot{Q}-\left(I_{z}-I_{x}\right) P R-I_{x y}(Q R+\dot{P})+I_{x z}\left(P^{2}-R^{2}\right)+I_{y z}(P Q-\dot{R}) \\
& \quad-S_{x}(\dot{W}+V P-U Q)+S_{z}(\dot{U}-V R+W Q)=M_{b} \\
& I_{z} \dot{R}-\left(I_{x}-I_{y}\right) P Q-I_{x y}\left(P^{2}-R^{2}\right)+I_{x z}(Q R-\dot{P})-I_{y z}(\dot{Q}+P R) \\
& \quad+S_{x}(\dot{V}+U R-W P)-S_{z}(\dot{U}+W Q-V R)=N_{b}
\end{aligned}
$$


However, it is assumed that the origin of the gravity coordinate system $O_{g} x_{g} y_{g} z_{g}$ is coincident with the origin $O_{b} x_{b} y_{b} z_{b}$ of the body coordinate system so $S_{x}=S_{y}=S_{z}=0$. The next assumption is that the missile is a body of revolution and has two planes of symmetry. Hence, all products of inertia are zero. The velocity vector of the missile in the $O_{n} x_{n} y_{n} z_{n}$ can be calculated as (Zipfel, 2007)

$$
\left[\begin{array}{c}
\dot{x}_{n} \\
\dot{y}_{n} \\
\dot{z}_{n}
\end{array}\right]=\left[\begin{array}{ccc}
e_{0}^{2}+e_{1}^{2}-e_{2}^{2}-e_{3}^{2} & 2\left(e_{1} e_{2}-e_{0} e_{3}\right) & 2\left(e_{0} e_{2}-e_{1} e_{3}\right) \\
2\left(e_{0} e_{3}-e_{1} e_{2}\right) & e_{0}^{2}-e_{1}^{2}+e_{2}^{2}-e_{3}^{2} & 2\left(e_{2} e_{3}-e_{0} e_{1}\right) \\
2\left(e_{1} e_{3}-e_{0} e_{2}\right) & 2\left(e_{0} e_{1}-e_{2} e_{3}\right) & e_{0}^{2}-e_{1}^{2}-e_{2}^{2}+e_{3}^{2}
\end{array}\right]\left[\begin{array}{c}
U \\
V \\
W
\end{array}\right]
$$

where $e_{0}, e_{1}, e_{2}, e_{3}$ - quaternion elements.

The integration of the rate of change of the quaternion vector is given as follows

$$
\left[\begin{array}{c}
\dot{e}_{0} \\
\dot{e}_{1} \\
\dot{e}_{2} \\
\dot{e}_{3}
\end{array}\right]=-\frac{1}{2}\left[\begin{array}{cccc}
0 & P & Q & R \\
-P & 0 & -R & Q \\
-Q & R & 0 & -P \\
-R & -Q & P & 0
\end{array}\right]\left[\begin{array}{c}
e_{0} \\
e_{1} \\
e_{2} \\
e_{3}
\end{array}\right]-k E\left[\begin{array}{c}
e_{0} \\
e_{1} \\
e_{2} \\
e_{3}
\end{array}\right]
$$

The gain $k$ drives the norm of the quaternion state vector to one. Next, the roll $\Phi$, pitch $\Theta$ and yaw $\Psi$ angles are calculated as below (Zipfel, 2007)

$$
\begin{aligned}
& \Phi=\arctan \frac{2\left(e_{0} e_{1}+e_{2} e_{3}\right)}{e_{0}^{2}-e_{1}^{2}-e_{2}^{2}+e_{3}^{2}} \quad \Theta=\arcsin \left[2\left(e_{0} e_{2}-e_{1} e_{3}\right)\right] \\
& \Psi=\arctan \frac{2\left(e_{0} e_{3}+e_{1} e_{2}\right)}{e_{0}^{2}+e_{1}^{2}-e_{2}^{2}-e_{3}^{2}}
\end{aligned}
$$

In missiles, the center of gravity normally shifts due to the burning off of the fuel. Forces $\mathbf{F}_{b}$ acting on the missile have been obtained by summing up the inertia (left hand side of the equation), gravity $\mathbf{F}_{G}$, aerodynamic $\mathbf{F}_{A}$, propulsion $\mathbf{F}_{P}$, control fins $\mathbf{F}_{C}$ and control loads from the lateral thrusters $\mathbf{F}_{T}$ (Yuhang et al., 2006)

$$
\mathbf{F}_{b}=\mathbf{F}_{G}+\mathbf{F}_{A}+\mathbf{F}_{P}+\mathbf{F}_{C}+\mathbf{F}_{T}
$$

In a similar way, the moments and the resultant moment $\mathbf{M}_{b}$ are calculated as follows

$$
\mathbf{M}_{b}=\mathbf{M}_{G}+\mathbf{M}_{A}+\mathbf{M}_{P}+\mathbf{M}_{C}+\mathbf{M}_{T}
$$

A diagram that shows the forces and moments acting on the missile is illustrated in Fig. 6 .

The vector of gravity loads acting on the object is calculated as

$$
\mathbf{F}_{G}=m g[-\sin \Theta, \cos \Theta \sin \Phi, \cos \Theta \cos \Phi]^{\mathrm{T}} \quad \mathbf{M}_{G}=[0,0,0]^{\mathrm{T}}
$$

The aerodynamic forces and moments in the $O_{b} x_{b} y_{b} z_{b}$ coordinate system are calculated as below

$$
\mathbf{F}_{A}=\frac{1}{2} \rho V_{0}^{2} S\left[-C_{X}, C_{Y},-C_{Z}\right]^{\mathrm{T}} \quad \mathbf{M}_{A}=\frac{1}{2} \rho V_{0}^{2} S d\left[-C_{L}, C_{M},-C_{N}\right]^{\mathrm{T}}
$$

where $\rho$ is the air density, $V_{0}$ - total flight velocity, $S$ - area of the missile cross section, $d$ - missile diameter and $C_{X}, C_{Y}, C_{Z}, C_{L}, C_{M}, C_{N}$ are force and moment coefficients, respectively. The aerodynamic forces, as given in the above equations, are functions of the aerodynamic coefficients which depend on factors such as the Mach number, the angle of attack, sideslip angle and angular velocities. The table lookup procedure has been used for obtaining the aerodynamic coefficients for various flow angles and the Mach numbers from an offline generated database. Aerodynamic characteristics of the missile have been determined using Arrow Tech PRODAS 


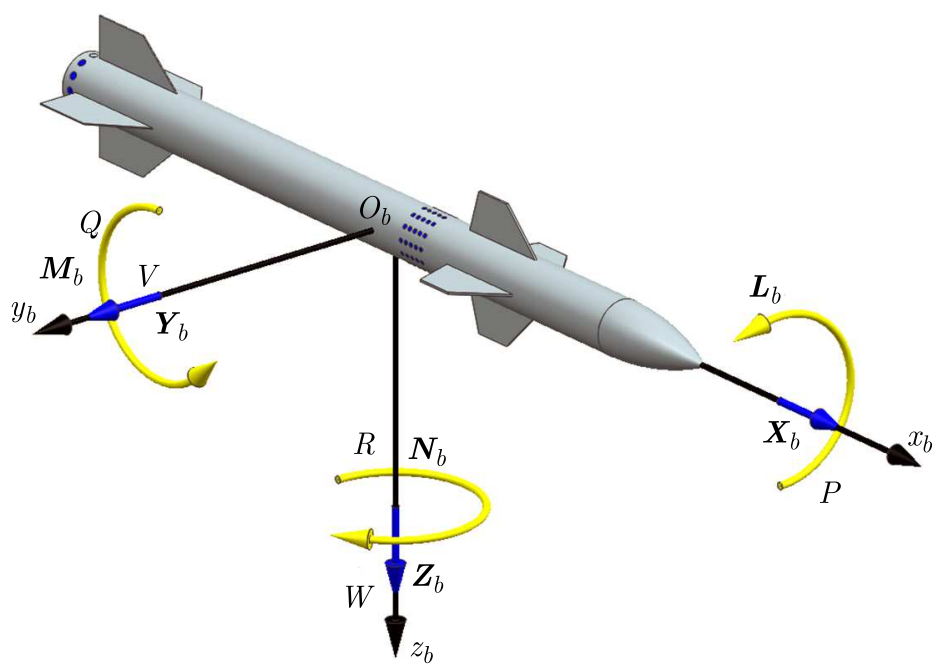

Fig. 6. Forces and moments acting on the missile

software (MAHER, 2002). The coefficients were also checked with the aim of Computational Fluid Dynamics (CFD) methods. Steady-state simulations for angles of attack in range from $-15^{\circ}$ to $15^{\circ}$ and Mach numbers (Ma) from 0.1 to 4 were performed to confirm the values of aerodynamic coefficients

$$
\begin{array}{ll}
C_{X}(\alpha, \beta, \mathrm{Ma})=C_{X 0}+C_{X \alpha 2} \sin ^{2} \alpha+C_{X \beta 2} \sin ^{2} \beta \\
C_{Y}(\beta, \mathrm{Ma})=C_{Y \beta} \sin \beta+C_{Y \beta 3} \sin ^{3} \beta & \\
C_{Z}(\alpha, \mathrm{Ma})=C_{Z \alpha} \sin \alpha+C_{Z \alpha 3} \sin ^{3} \alpha & C_{L}(\mathrm{Ma})=C_{L P} \frac{P d}{2 V_{0}} \\
C_{M}(\alpha, \mathrm{Ma})=C_{M \alpha} \sin \alpha+\frac{Q d}{2 V_{0}} C_{M Q} & C_{N}(\beta, \mathrm{Ma})=C_{N \beta} \sin \beta+\frac{R d}{2 V_{0}} C_{N R}
\end{array}
$$

where $C_{L P}$ is rolling moment coefficient derivative with the roll rate, $C_{M Q}$ - pitching moment coefficient derivative with the pitch rate, $C_{N R}$ - yawing moment coefficient derivative with the yaw rate.

Some of the coefficients are shown in Fig. 7.

(a)

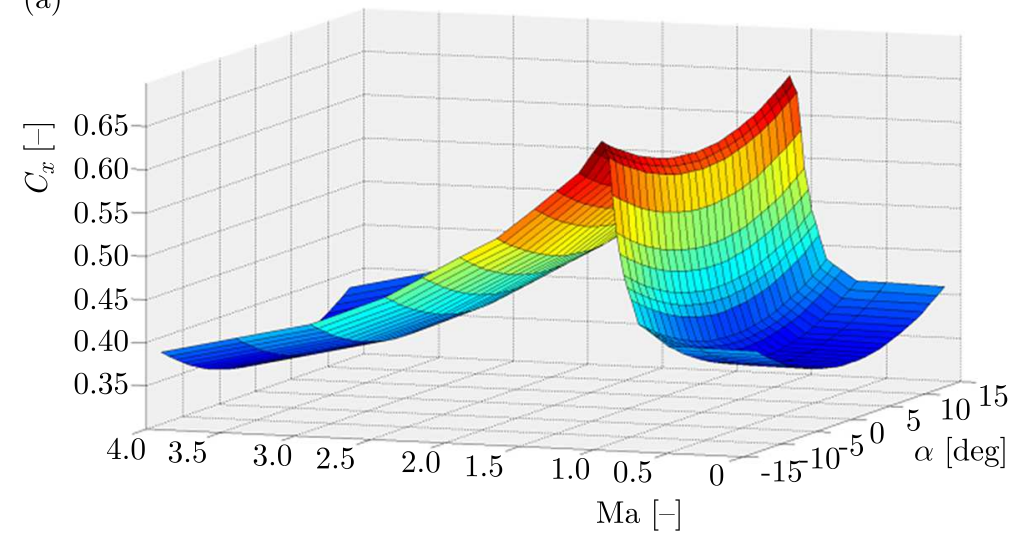

(b)

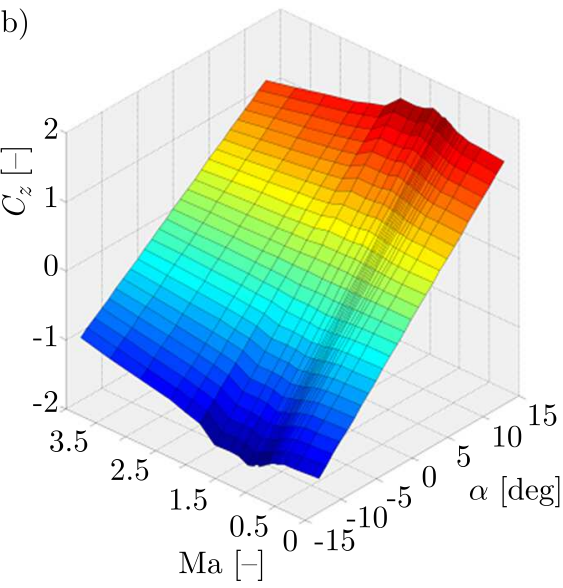

Fig. 7. (a) Axial and (b) normal force coefficients as a function of the Mach number and angle of attack

The International Civil Aviation Organization (ICAO) standard atmosphere model has been used as the flight environment (Zarchan, 2012). Propulsive loads were calculated as follows

$$
\mathbf{F}_{P}=[T(t), 0,0]^{\mathrm{T}} \quad \mathbf{M}_{P}=[0,0,0]^{\mathrm{T}}
$$


The thrust profile is a known function of time $T(t)$ and was obtained on the engine test stand. It was assumed that main engine thrust force was parallel to the $O_{b} x_{b}$ axis the body coordinate system. Control forces generated by fin deflections are defined as follows

$$
\begin{aligned}
& \mathbf{F}_{C}=\frac{1}{2} \rho V_{0}^{2} S\left[\begin{array}{c}
X_{\delta_{A}} \delta_{A}+X_{\delta_{B}} \delta_{B}+X_{\delta_{C}} \delta_{C}+X_{\delta_{D}} \delta_{D} \\
Y_{\delta_{A}} \delta_{A}+Y_{\delta_{B}} \delta_{B}+Y_{\delta_{C}} \delta_{C}+Y_{\delta_{D}} \delta_{D} \\
Z_{\delta_{A}} \delta_{A}+Z_{\delta_{B}} \delta_{B}+Z_{\delta_{C}} \delta_{C}+Z_{\delta_{D}} \delta_{D}
\end{array}\right] \\
& \mathbf{M}_{C}=\frac{1}{2} \rho V_{0}^{2} S d\left[\begin{array}{c}
L_{\delta_{A}} \delta_{A}+L_{\delta_{B}} \delta_{B}+L_{\delta_{C}} \delta_{C}+L_{\delta_{D}} \delta_{D} \\
M_{\delta_{A}} \delta_{A}+M_{\delta_{B}} \delta_{B}+M_{\delta_{C}} \delta_{C}+M_{\delta_{D}} \delta_{D} \\
N_{\delta_{A}} \delta_{A}+N_{\delta_{B}} \delta_{B}+N_{\delta_{C}} \delta_{C}+N_{\delta_{D}} \delta_{D}
\end{array}\right]
\end{aligned}
$$

where $\delta_{A}, \delta_{B}, \delta_{C}, \delta_{D}$ are the control surfaces deflection angles. Next, the control forces generated by the reaction control system are calculated (DeSpirito, 2013). The missile has a set of small solid fuel engines placed at the end of the body. It is assumed that there are $N=12$ jets. It is assumed that nozzles of these engines are perpendicular to the longitudinal axis of symmetry of the missile (Weinacht, 2004). The jets are translated by the vector $\mathbf{r}_{T i}=\left[x_{T i}, y_{T i}, z_{T i}\right]$ from the origin of the $O_{b} x_{b} y_{b} z_{b}$ coordinate system, where $i=1, \ldots, N$ is the number of the jet engine (Zhen et al., 2012).

(a)

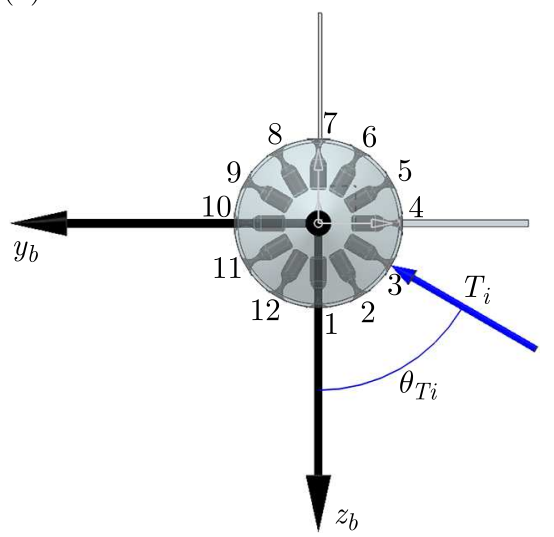

(b)

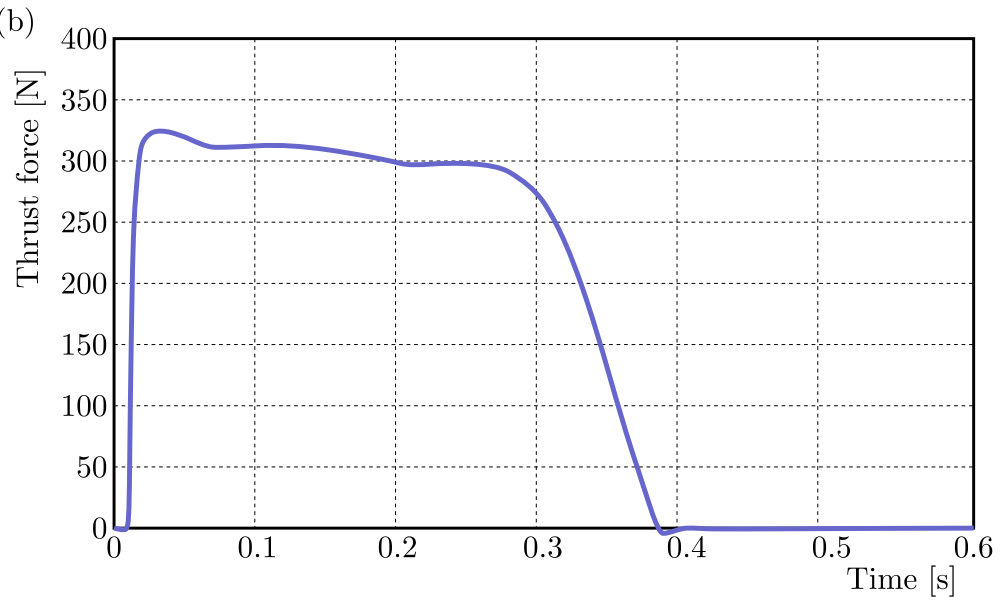

Fig. 8. (a) Reaction control system which is simulated, (b) the jet engine thrust curve

The force from the $i$-th jet engine in the $O_{b} x_{b} y_{b} z_{b}$ coordinate system is described as below

$$
\mathbf{F}_{T i}=T_{i}\left[0, \sin \theta_{T i},-\cos \theta_{T i}\right]^{\mathrm{T}}
$$

The moments generated by this pulse jet are

$$
\mathbf{M}_{T i}=\mathbf{r}_{T i} \times \mathbf{F}_{T i}=T_{i}\left[-y_{T i} \cos \theta_{T i}-z_{T i} \sin \theta_{T i}, x_{T i} \cos \theta_{T i}, x_{T i} \sin \theta_{T i}\right]^{\mathrm{T}}
$$

It is assumed that the jet engine thrust force (Fig. 8) is a function of time (Fenghua et al., 2008). The shape of the curve has been designed with the aim of the method which was presented in (Fleeman, 2006). The maximum thrust and jet engine size is limited also by the diameter of the missile. There are defined some time constants which describe delays between subsequent phases of the jet firing logic: $\tau_{1}$ - start time for the first jet engine, $\tau_{2}-$ burnout time for the first engine, $\tau_{3}$ - start time for the second jet engine, $\tau_{4}$ - burnout time for the second engine, $\tau_{5}$ - main engine launch time. These time constants are connected with two specific pitch angle values: $\theta_{\tau 2}$ - pitch angle after the first jet engine burnout, $\theta_{\tau 4}$ - pitch angle after the first jet engine burnout. These parameters have been calculated offline with the aim of optimisation methods and implemented in the simulation. The methods by which the time constants have 
been calculated are out of the scope of this article. The described mathematical model has been implemented in the Matlab software. The inputs to the model are the launch conditions, while the outputs are the missile flight data (velocity, acceleration, etc.).

\section{Simulation results}

In this part, simulation results are presented. The missile is controlled by twelve small engines mounted at the end of the missile tail. As the missile is launched, one of the engines is ignited to orient the object in the demanded direction. Next, the second pulse engine is used to counteract the pitch motion and, finally, the main missile engine is started. The key goals of the simulation are to find control forces of the lateral jets, time constants and the best height at which the missile should be ejected.

In the first test, the dependency between the $\tau_{3}$ time constant and the final pitch angle has been analyzed. The second pulse engine was ignited with various delays. In Fig. 9, the missile flight trajectories for various $\tau_{3}$ time constants are presented. The burntime of the single pulse jet was $0.37 \mathrm{~s}$ and the thrust force was assumed constant and equal to $313 \mathrm{~N}$. The missile was ejected vertically (initial pitch angle $90^{\circ}$ ) with the velocity $25 \mathrm{~m} / \mathrm{s}$. Before the launch, the missile was located in the origin of the inertial coordinate system $O_{n} x_{n} y_{n} z_{n}$.

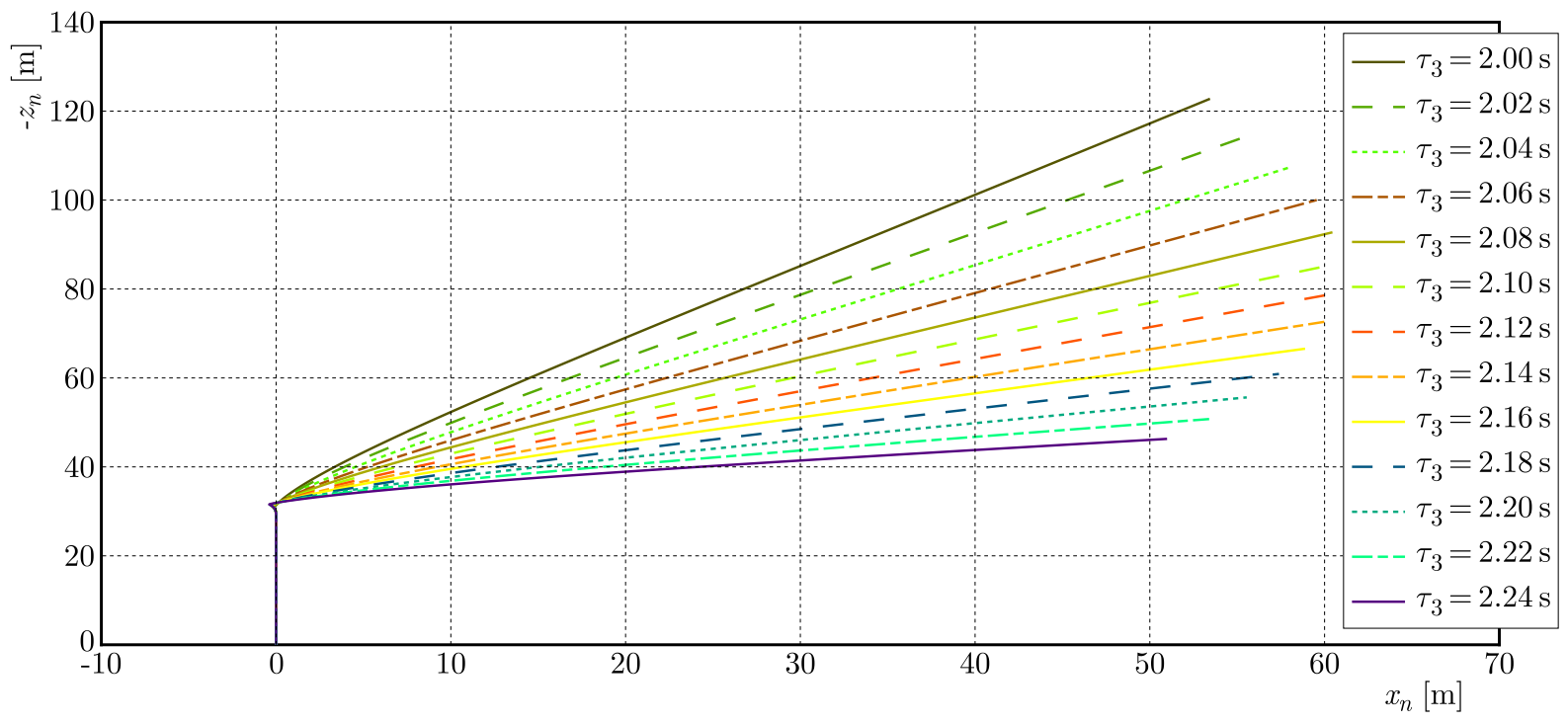

Fig. 9. Trajectories as a function of $\tau_{3}$ time constants

The time constant $\tau_{3}$ was varied from $2 \mathrm{~s}$ to $2.24 \mathrm{~s}$. For the smallest value of this time constant, the pitch angle was about nearly $60^{\circ}$ (for vertically oriented missile pitch angle $90^{\circ}$ ). It is planned to use the inertial measurement unit to measure the actual pitch angle. For the biggest value of this time constant, the pitch angle is about $10^{\circ}$. The main conclusion from this example is that with using of this time constant it is possible to achieve various pitch angles.

In Fig. 10, the pitch angular velocity and the pitch angle as a function of time and for various time constants which were used during simulations are presented.

The angular velocity does not change until $1.8 \mathrm{~s}$ because during this time no pulse jet is used. Next, the first pulse jet is ignited and the missile pitch velocity changes rapidly as expected. Later, the second jet engine is launched and the angular velocity decreases nearly to zero. After $2.3 \mathrm{~s}$ there occurs a very small positive singular velocity. It is difficult to drive this small difference to zero because the first and second lateral jet thrust curves are the same. The bigger the $\tau_{3}$ time constant, the smaller pitch angle is achieved at the end of the simulation. 


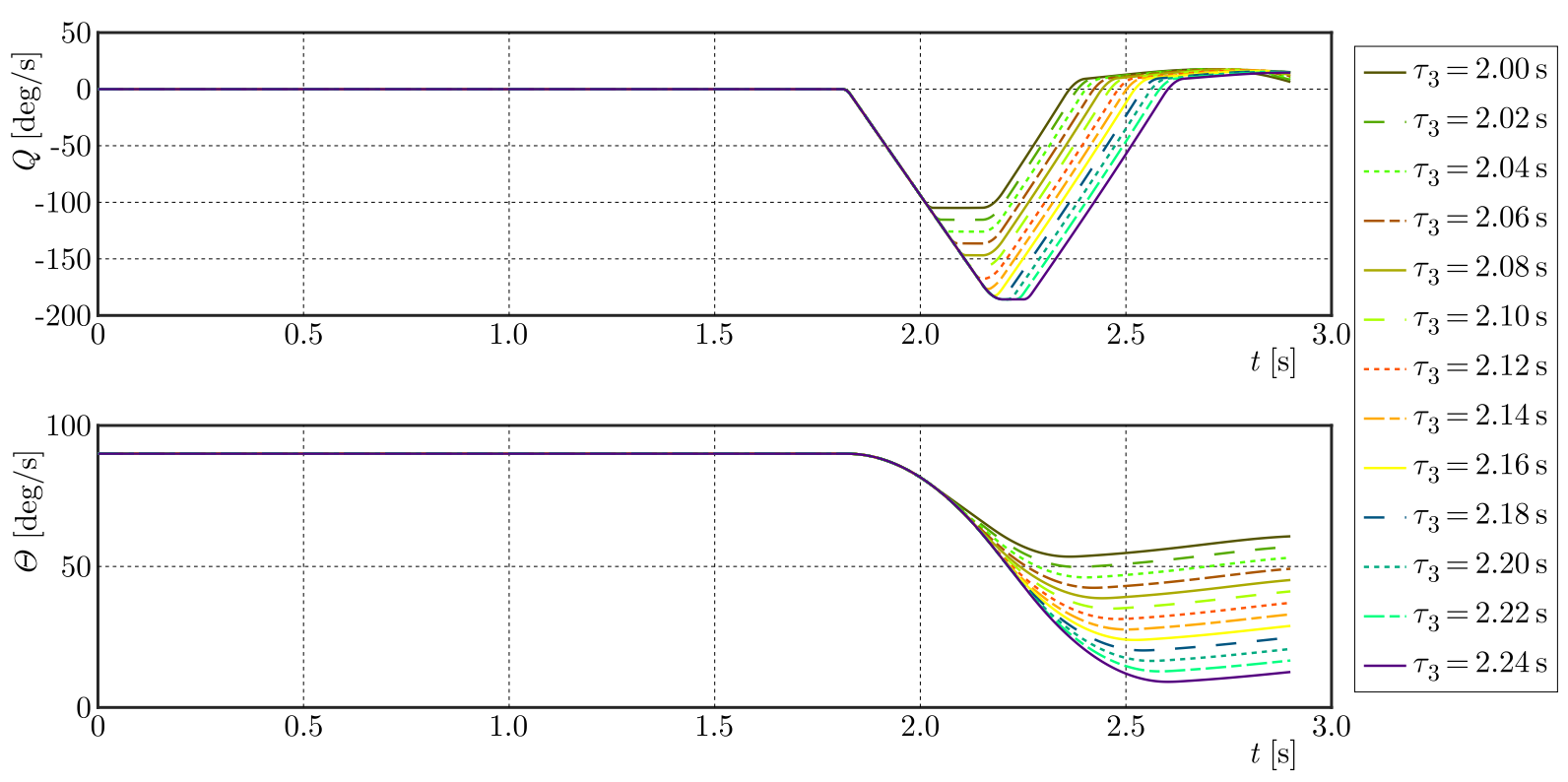

Fig. 10. Pitch velocities and pitch angles for various $\tau_{3}$ time constants

Next, the influence of $\tau_{1}$ on the missile altitude has been tested. In Fig. 11, flight paths for various $\tau_{1}$ are shown. The launch velocity has been assumed to be equal to $27 \mathrm{~m} / \mathrm{s}$.

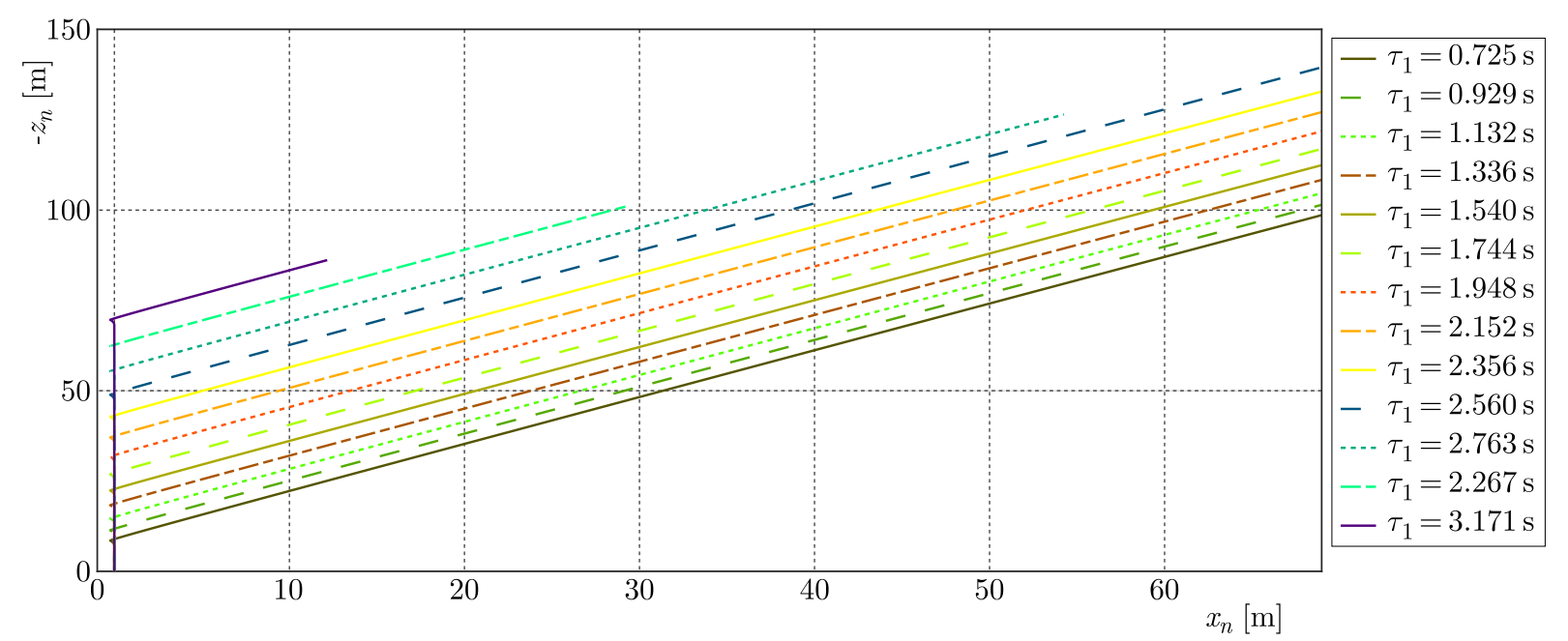

Fig. 11. Flight trajectories for various $\tau_{1}$ time constants

The time constants $\tau_{2}, \tau_{3}, \tau_{4}$ and $\tau_{5}$ are connected with the missile launch $\tau_{1}$. Satisfactory results of these simulations have been obtained.

In Fig. 12, the pitch angular velocity and the pitch angle as a function of the first delay $\tau_{1}$ are shown.

At the end of the simulation, the pitch angle is about $53^{\circ}$. The total maneuver time does not exceed $0.6 \mathrm{~s}$, which is quite a small value.

The other goal of the experiments was to compare the simulation results for the object with the aerodynamic control surfaces only and the object with the gasdynamics controls (Fig. 13). In the simulations, two objects are launched vertically and then they are aiming to the same point. 


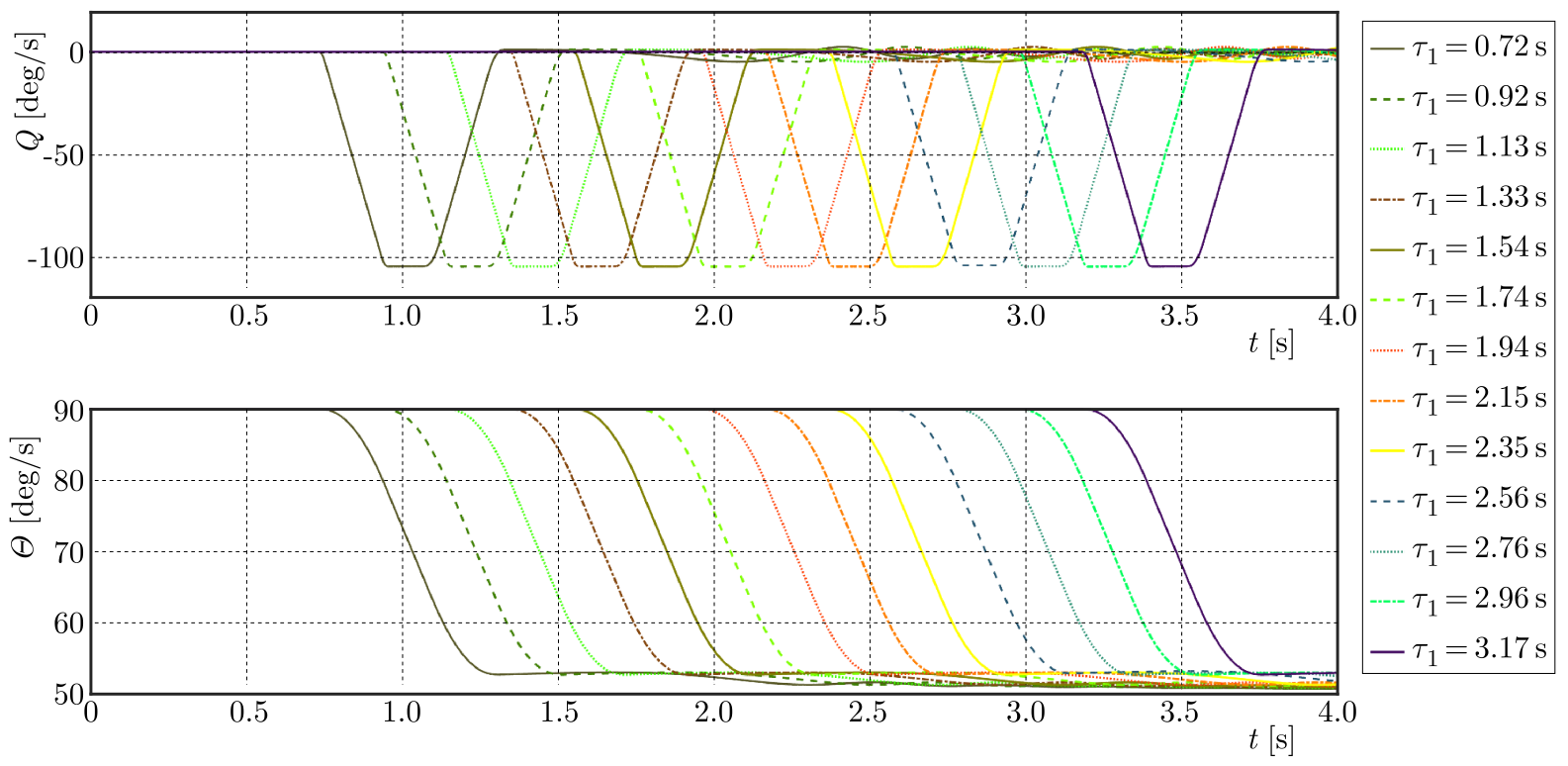

Fig. 12. Missile angular pitch velocity and pitch angle as a function of time

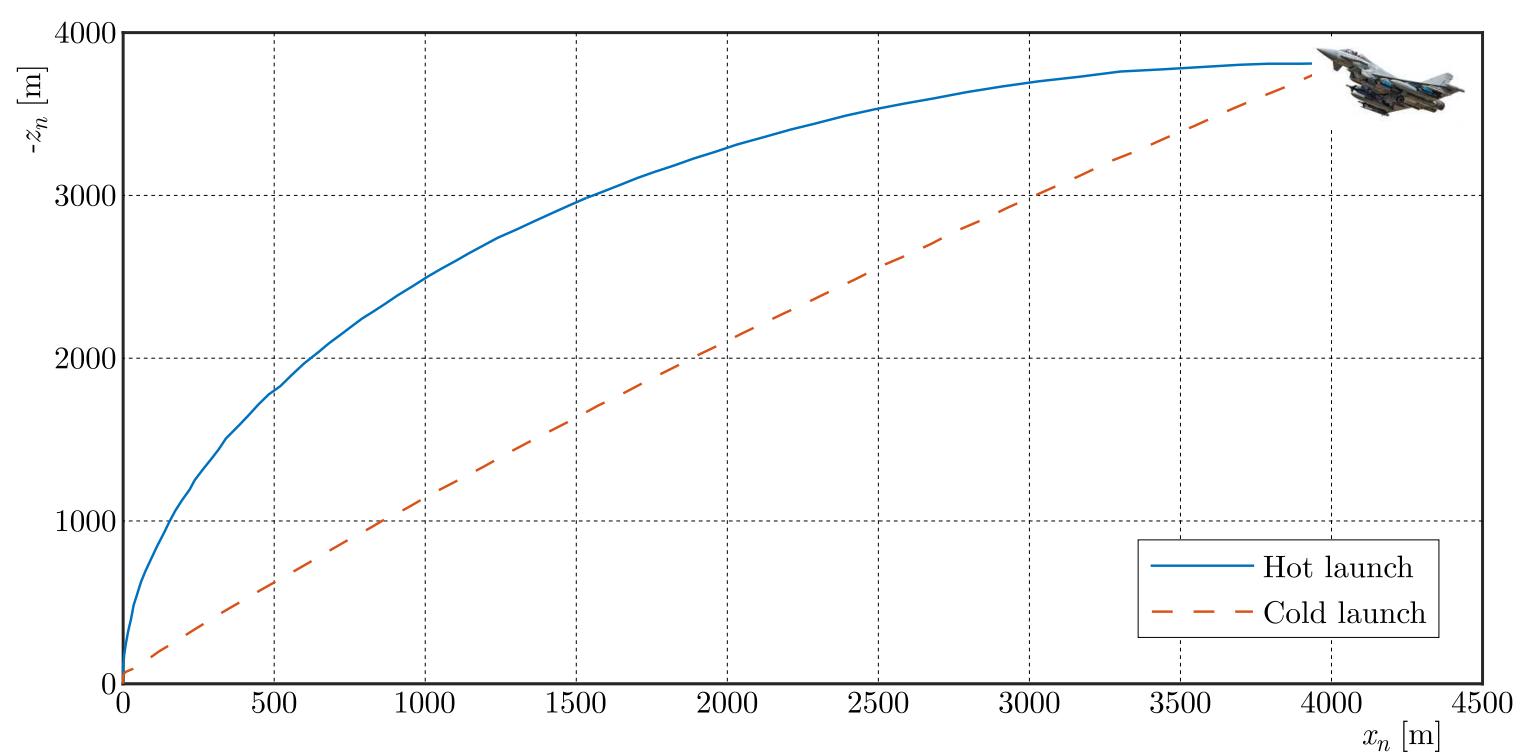

Fig. 13. Comparison of the trajectories for cold and hot launched missiles

There is only motion in the vertical plane, so $y$ distance is zero. There is a significant difference between both curves. The object with gasdynamics control can move along a straight line, which is much more effective than flight along a parabolic curve. the missile with the aerodynamic control has not those capabilities. The missile with the gasdynamics control is able to reach the control point at a different trajectory and also can hit targets which are very low. The missile with the gasdynamic control is able to reach the target in a shorter time than the missile with the classical vertical hot launch system. The presented method is able to save about $14 \%$ of fuel. The control system model used in the simulation is adequate to this type of the object and is able to control precisely this object. The object can rotate in the air because there is small damping in the air. When the delay is too big, the object can rotate too much and hits the ground. The smulation results show capabilities of the presented control method. 


\section{Conclusion}

This article investigates the dynamic characteristics of the cold launched missile with the gasdynamics control. The soft launch ignites the rocket motor after the missile has been launched and directed towards the target. Analysis and simulation have been conducted to investigate the dynamics of the missile. The missile model has been implemented in Simulink software. Numerical experiments have shown some advantages of the proposed method like higher range. It has been obtained that it is possible to use the gasdynamic control to orient the missile objects during the launch phase. It can be concluded that the presented model of the missile launch phase works properly.

\section{References}

1. Baranowski L., 2013, Numerical testing of flight stability of spin-stabilized artillery projectiles, Journal of Theoretical and Applied Mechanics, 51, 2, 375-385

2. Blakelock J., 1991, Automatic Control of Aircraft and Missiles, New York, NY, John Wiley \& Sons, Inc.

3. Despirito J., 2013, Lateral Reaction Jet Flow Interaction Effects on a Generic Fin-Stabilized Munition in Supersonic Crossflow, Army Research Laboratory

4. Fenghua H., Kemao M., Yu Y., 2008, Firing logic optimization design of lateral jets in missile attitude control systems, 17th IEEE International Conference on Control Applications, San Antonio

5. Fleeman E., 2006, Missile Design and System Engineering, AIAA Distinguished Lecture

6. Maher K., 2002, PRODAS V3 - Technical Manual, Arrow Tech

7. MBDA Systems, 2014, MBDA - Common Anti-air Modular Missile (CAMM), Retrieved from https://youtu.be/Nnb20mrT1kw

8. TOR-M1 9A331 (SA-15 Gauntlet) surface-to-air missile, 2012, Retrieved from https://www.youtube.com/watch?v=qcC0rXxXVNA

9. Weinacht P., 2004, Lateral Control Jet Aerodynamic Predictions for a 2.75-in Rocket Testbed Munition, Army Research Laboratory

10. Yuhang W., Yu Y., Kemao M., 2006, Lateral thrust and aerodynamics blended control system fesign based on variable structure model following, Intelligent Control and Automation, Dalian, $8183-8186$

11. Zarchan P., 2012, Tactical and Strategic Missile Guidance, American Institute of Aeronautics and Astronautics

12. Zhen S., Wenqiao M., Yufang Z., Huichao H., 2012, Lateral thrust and aerodynamics compound control system of missile based on adaptive fuzzy control, Computational Intelligence and Design (ISCID), Hangzhou

13. Zipfel P., 2007, Modeling and Simulation for Aerospace Vehicle Dynamics, American Institute of Aeronautics and Astronautics

14. Żugaj M., GŁęBocki R., 2010, Model of gasodynamic control system for guided bombs, Journal of Theoretical and Applied Mechanics, 48, 1, 27-44 